\title{
BMJ Open Study protocol of a multicentre cohort pilot study implementing an expanded preconception carrier-screening programme in metropolitan and regional Western Australia
}

Royston Ong, ${ }^{\oplus 1,2}$ Samantha Edwards, ${ }^{1,2}$ Denise Howting, ${ }^{1,2}$ Benjamin Kamien, ${ }^{3}$ Karen Harrop, ${ }^{3}$ Gianina Ravenscroft, ${ }^{1,2}$ Mark Davis, ${ }^{4}$ Michael Fietz, ${ }^{4,5}$ Nicholas Pachter, ${ }^{3}$ John Beilby, ${ }^{4}$ Nigel Laing ${ }^{1,2}$

To cite: Ong R, Edwards S, Howting D, et al. Study protocol of a multicentre cohort pilot study implementing an expanded preconception carrier-screening programme in metropolitan and regional Western Australia. BMJ Open 2019;9:e028209. doi:10.1136/ bmjopen-2018-028209

- Prepublication history and additional material for this paper are available online. To view these files, please visit the journal online (http://dx.doi. org/10.1136/bmjopen-2018028209).

Received 27 November 2018 Revised 5 May 2019 Accepted 3 June 2019

Check for updates

(C) Author(s) (or their employer(s)) 2019. Re-use permitted under CC BY-NC. No commercial re-use. See rights and permissions. Published by BMJ.

For numbered affiliations see end of article.

Correspondence to

Professor Nigel Laing;

nigel.laing@perkins.uwa.edu.au

\section{ABSTRACT}

Introduction Preconception carrier screening (PCS)

identifies couples at risk of having children with recessive genetic conditions. New technologies have enabled affordable sequencing for multiple disorders simultaneously, including identifying carrier status for many recessive diseases. The aim of the study was to identify the most effective way of delivering PCS in Western Australia (WA) through the public health system. Methods and analysis This is a multicentre cohort pilot study of 250 couples who have used PCS, conducted at three sites: (1) Genetic Services of Western Australia, (2) a private genetic counselling practice in Perth and (3) participating general practice group practices in the Busselton region of WA. The primary outcome of the pilot study was to evaluate the feasibility of implementing the comprehensive PCS programme in the WA healthcare system. Secondary outcome measures included evaluation of the psychosocial impact of couples, such as reproductive autonomy; identification of areas within the health system that had difficulties in implementing the programme and evaluation of tools developed during the study.

Ethics and dissemination Approval was provided by the Women and Newborn Health Service Human Research Ethics Committee (HREC) at King Edward Memorial Hospital for Women (RGS0000000946) and the University of Western Australia (UWA) HREC (RA/4/20/4258). Participants may choose to withdraw at any time. Withdrawal will in no way affect participating couples' medical care. Study couples will be redirected to another participating health professional for consultation or counselling in the event of a health professional withdrawing. All evaluation data will be deidentified and stored in a password-protected database in UWA. In addition, all hard copy data collected will be kept in a locked cabinet within a secure building. All electronic data will be stored in a password-protected, backed-up location in the UWA Institutional Research Data Store. All evaluative results will be published as separate manuscripts, and selected results will be presented at conferences.

\section{Strengths and limitation of this study}

- Participation of various service providers, such as Genetic Services of Western Australia (GSWA), will allow the research team to understand limitations in infrastructure and bottlenecks in integrating preconception carrier screening (PCS) into the Western Australia (WA) public health system.

- The study will evaluate stakeholders' experiences to understand different requirements between metropolitan and regional areas.

- Evaluating training materials and tools developed during the study will allow refinements and improvements for future implementation.

- The small study will likely identify only major issues encountered in different settings within WA. Other limitations may include individuals wishing to receive individual carrier reports rather than a couple report.

- Most couples recruited through GSWA or the private genetic counselling practice may present a self-selection bias, which may influence measurements of uptake rates or some psychosocial evaluation, including motivations for PCS.

\section{INTRODUCTION}

Preconception carrier screening (PCS) involves screening individuals or couples for recessive mutations. These couples do not usually have an a priori increased risk of being a carrier based on their or their partners' personal or family disease history. The aim of PCS was therefore not for early diagnosis/ prevention and treatment but to facilitate reproductive decision-making. ${ }^{1}$

Recently, the American College of Obstetricians and Gynaecologists recommended that 'each obstetrician-gynecologist or other health care provider or practice should establish a standard approach (to carrier 
screening) that is consistently offered to and discussed with each patient, ideally before pregnancy'. ${ }^{2}$

As a result of falling sequencing costs and increased cost-effectiveness of sequencing technologies, ${ }^{3}{ }^{4}$ pilot studies have been suggested as a means to research: (1) implementing PCS, including which genes to screen; (2) population and health practitioner attitudes to screening; (3) counselling requirements and (4) laboratory infrastructure requirements. ${ }^{5}$ It has also been proposed that pilot studies should be carried out in multiple countries ${ }^{6}$ since best practice PCS will vary, depending on each country's health system.

In recent years, some countries have begun researching implementation of pan-ethnic expanded PCS programmes into their health systems. ${ }^{78}$ For example, a gene panel that covers 50 serious, early-onset, autosomal-recessive diseases was developed in the Netherlands, ${ }^{7}$ while Belgium has been actively working towards implementing an expanded carrier-screening programme into its healthcare system. ${ }^{8}$

Australia lags behind world best practice in PCS and what is available is highly variable in different States. All available services are consumer-paid, including tests offered by commercial entities. In Victoria, for example, the Victorian Clinical Genetics Service offers the Prepair screening programme for three common recessive diseases: cystic fibrosis, spinal muscular atrophy and fragile $\mathrm{X}$ syndrome, and PCS is being offered through some general practitioner (GP) group practices providing a route to access the commercial programmes. In Western Australia (WA), carrier screening is generally only performed as cascade screening for families with a history of recessive disease. The piecemeal nature of carrier screening in Australia means that there are inequities in access and outcomes across states and populations.

Our research in WA shows that there is considerable support for PCS. Two-thirds of nearly 1000 Western Australians surveyed said they would use PCS if it were available to them. ${ }^{9}$ Of those who said they would use PCS, $80 \%$ said they would like to access the test and obtain results from their GP, and $40 \%$ said they would like to do so through a genetic counsellor (GC). This clearly indicates that there is an appetite for PCS in the WA community, as well as suggests possible delivery methods we can evaluate. ${ }^{9}$ However, it is well known that intentions to do a behaviour may differ from actual participation and may be influenced by different factors. ${ }^{10}$

This study protocol seeks to identify the most effective way of delivering PCS in WA, given that WA spans over 2.5 million square kilometres. To do this, we are leveraging existing research and health infrastructures, including GSWA, PathWest Laboratory Medicine (PathWest) in the Department of Health and the Busselton Population Medical Research Institute (BPMRI). In addition, we will explore the role GPs and private genetic counselling clinics can play in providing PCS in the metropolitan region.

\section{METHOD}

\section{Study design}

This is a multicentre cohort study offering PCS to couples planning on starting or extending a family (see online supplementary figure 1). The study will be conducted through three routes: GSWA, a private genetic counselling practice in the metropolitan area and participating general practices in Busselton, WA. Recruitment for the study started in early September 2018 and will continue for 2 years or until study numbers are reached.

\section{Patient and public involvement}

The study is advised by the Western Australian Health Translation Network Consumer and Community Health Research Network from the beginning. The study will have two 'research buddies' from the Network throughout the study. The research buddies are critical to ensure that all communications with the public, including study results, the video tool and any print material produced, are easily understood.

\section{Metropolitan region}

\section{Genetic Services of Western Australia (GSWA)}

GSWA provides genetic counselling for individuals and couples with a family history of a genetic condition. In this study, GSWA will recruit such couples who are planning more children, which will enable us to determine specific requirements necessary for PCS for this subset of couples.

\section{Private genetic counselling practice}

As mentioned previously, in WA, carrier screening is generally only performed for families with a history of recessive disease. Therefore, couples without a family history of recessive diseases and are interested in PCS will have to visit a private genetic counselling practice offering commercial PCS panels to access the test. This recruitment arm will provide the study an opportunity to recruit couples from the metropolitan area who wish to be screened for possible genetic conditions, but who do not have a family history of a genetic disease.

The GG will offer the research test protocol as an option, in addition to the testing from commercial suppliers currently made available. Participants will pay for any pretest consult as per the GC's rates.

If a couple chooses the research protocol, the laboratory testing will be free of charge to the couple.

\section{Regional Areas}

\section{GP practices in the Busselton region}

The Busselton community and their GPs have a 50-year history of participation in research studies. ${ }^{11}$ The voluntary participation rate in BPMRI projects is very high. Participating GP practices will recruit couples that are planning to have children. Recruiting couples in the Busselton region will enable us to determine the specific requirements necessary for successful implementation of PCS in regional communities. The BPMRI will facilitate 
dissemination of awareness of the study in the Busselton region.

\section{Procedure}

Participant recruitment

We aimed to recruit 250 couples (500 individuals) between the metropolitan and Busselton sites.

\section{Metropolitan region (GCs)}

The aim was to recruit 100 couples in the metropolitan region.

Posters and leaflets will be placed in GSWA and the private genetic counselling practices to raise awareness about the study and to prompt potential participants to ask about the study during their visits. Potential participant couples will also be made aware of the study through GCs and clinical geneticists during clinic visits.

Potential participants will be given a study information pack, consisting of the patient information sheet and consent form, to take home and consider with their partner at their leisure.

Couples interested in the study will have to return for couple-based pretest counselling. If the couple decides to participate in the study, they will sign the consent form. Couples will then be asked if they are willing to participate in the evaluation of the PCS programme to inform researchers about their experience and how the programme can be improved.

If potential participants decide not to participate, they can voice their reasons through an online survey link provided in the leaflet.

Couples will be reminded that that they can withdraw from the study at any time.

\section{Busselton region (GP practices)}

The aim was to recruit 150 couples in the Busselton region. Posters and leaflets will be placed in each participating GP clinic and at the BPMRI, to raise awareness about the study. In addition, newspaper articles and other media will be used to increase awareness of the study.

Potential eligible participants will also be made aware of the study during GP visits. Interested potential participants will be given a study information pack, including the patient information sheet and consent form and study pamphlet, to take home and consider with their partner, at their leisure.

Potential participants interested in the study will return for couple-based pretest counselling provided by their GP. If the couples decide to participate in the study, they will sign the consent form. Couples will also be asked if they are willing to participate in the evaluation of the PCS programme to inform researchers about their experience and how the programme can be improved.

If potential participants decide not to participate, they can voice their opinions through an online survey provided in the leaflet.

Couples will be reminded that they can withdraw from the study at any time.

\section{Participant inclusion criteria}

Each participating couple must meet the following requirements to be enrolled in this study:

1. Not be pregnant at the time of recruitment but considering having children in the future.

2. Couples must participate in the study together.

3. Both members of the couples must be at least 18 years of age.

4. Couples who have had a pregnancy loss or a child with a serious genetic condition who are planning more children or who have a family history of a genetic disorder (only applicable to GSWA).

\section{Participant exclusion criteria}

Couples meeting any of the following criteria will be excluded from the study:

1. Are pregnant at the time of recruitment.

2. Only one member of the couple agrees to participate in the study.

3. The couple (or one of them) are younger than 18 years of age.

4. Have not had a pregnancy loss, or a child with a serious genetic condition, or a family history of a genetic disorder (only applicable to GSWA).

5. Same sex couples.

Health professional recruitment

Metropolitan region (GCS)

An email inviting potential participating GCs and/ or clinical geneticists to an information session will be sent to GSWA staff and the private GC prior to the session. The session will inform GCs and clinical geneticists about the study, recruitment criteria and reporting methods and questions pertaining to the study will be clarified.

Any health professional that expresses an interest will be given a health professional information pack, consisting of an information sheet and a consent form, to take away to deliberate. Signed consent forms can either be collected by the study GC or sent via a stamped addressed envelope to the study principal investigator (PI).

\section{Busselton (regional GP practices)}

An email inviting potential participating GPs to an information session with the study GC and the PI will be sent to all GPs within the Busselton region. The BPMRI will facilitate recruitment of Busselton GPs. The session will inform GPs about the study, recruitment criteria, training involved and reporting methods and questions pertaining to the study will be clarified.

Any GP who expresses an interest in participating will be given a health professional information pack, consisting of an information sheet and a consent form, to take away to consider. Signed consent forms can be sent via a stamped addressed envelope to the study PI.

Participating GPs will be trained by the study GC to provide pretest counselling to potential participant couples. 


\section{Biospecimen collection}

For all sites, following appropriate informed consent, $4 \mathrm{~mL}$ of venous blood will be collected at any PathWest collection centre.

Collected blood will be sent to the Department of Diagnostic Genomics, PathWest, QEII Medical Centre, for DNA extraction and storage. The DNA will be handled, prepared and sequenced within the Department of Diagnostic Genomics, which has been accredited by the National Association of Testing Authorities (NATA) for massively parallel sequencing (MPS).

\section{MPS and variant curation}

DNA will be sequenced using a custom enrichment capture panel, which was developed by the research team and synthesised by Illumina. The panel consisting of 474 genes associated with 440 childhood and infant lethal and/or debilitating recessive diseases fulfilled the current recommendations that genes included in a PCS panel should cause life-limiting diseases and/or diseases that cause physical or mental impairment. ${ }^{2}{ }^{12}$ Sequence data will be mapped, annotated and interrogated with Alissa Interpret (Agilent Technologies), as used routinely in the Department of Diagnostic Genomics, PathWest.

Only pathogenic or likely pathogenic (American College of Medical Genetics guidelines: classes 4 and $5)^{13}$ recessive variants identified in the same gene in both members of a couple or identified on the X chromosome in the female partner will be reported.

\section{Quality control and quality assurance}

All methods will be conducted and results will be analysed according to NATA-accredited protocols. Senior scientists-in-charge at PathWest will have responsibility for the laboratory data quality control.

The head of the Department of Diagnostic Genomics, PathWest, will address any quality control issues.

\section{Reporting}

Given the large number of genes tested in expanded PCS, individual post-test counselling will be extremely time consuming. ${ }^{14}$ Therefore, it has been suggested that post-test counselling should only be offered to couples who were identified to have pathogenic variants in the same gene ('high-risk' couples). ${ }^{12}$ Thus, results will be analysed and reported on a couple basis, and a couple will be issued with one of two reports, either 'high-risk' or 'low-risk'. In addition, as requested by our institution's human ethics committee, if couples agree to participate in the study, but choose not to receive any results, this option is available in the consent form. No results will be communicated to such couples.

\section{High-risk couples}

PathWest will generate a high-risk report if class 4 or 5 variants are identified in the same gene in both partners, or in an X-linked gene in the female partner. Following consultation with the clinical genetics team at GSWA, the study GC will deliver high-risk results, including appropriate preliminary information over the telephone. The couple is then referred to GSWA for more detailed genetic counselling regarding the implications of their high-risk finding and what reproductive options are available to them. A copy of the report and referral will be forwarded to the recruiting health professional.

'Low-risk' couples

PathWest will notify the study GC for low-risk couples, that is, those with no pathogenic recessive variants identified in the same gene. Low-risk couples will receive a letter generated by the study GC outlining their result and providing contact details of the study GC for further clarification if needed. The referring clinician will receive a copy.

A low-risk result means that the couple's risk of having a child with a severe recessive condition among those screened for by the PCS test has been significantly reduced.

\section{Quality control and quality assurance}

GSWA clinical geneticists will be in charge of ensuring consistency in counselling provided for high-risk couples and will address any counselling issues.

\section{STUDY AIMS AND OBJECTIVES \\ Aims}

The aim of the study was to identify the most effective way of delivering PCS in WA through the public health system.

\section{Objectives}

Our objectives of the study were

1. To perform a pilot study of PCS in WA using the proposed protocol.

2. To evaluate the effect of this protocol on the reproductive autonomy of couples who participated in the study.

3. To evaluate the effectiveness of the tools developed during the study.

4. To refine the initial protocol based on the evaluation of the protocol until the protocol is optimised to one that can be implemented.

\section{Outcomes}

The outcomes of the project were as follows:

1. Testing of a comprehensive PCS programme that we believe has the potential to be successful in the context of the Western Australian health system.

2. Evaluation of the effect of the proposed protocol on the reproductive autonomy of participating couples and the effectiveness of the tools developed in the study.

3. Identification of issues with the protocol.

4. Improvement of the protocol to one that will work better in the Western Australian health system and hopefully one that could be implemented in the WA health system. 


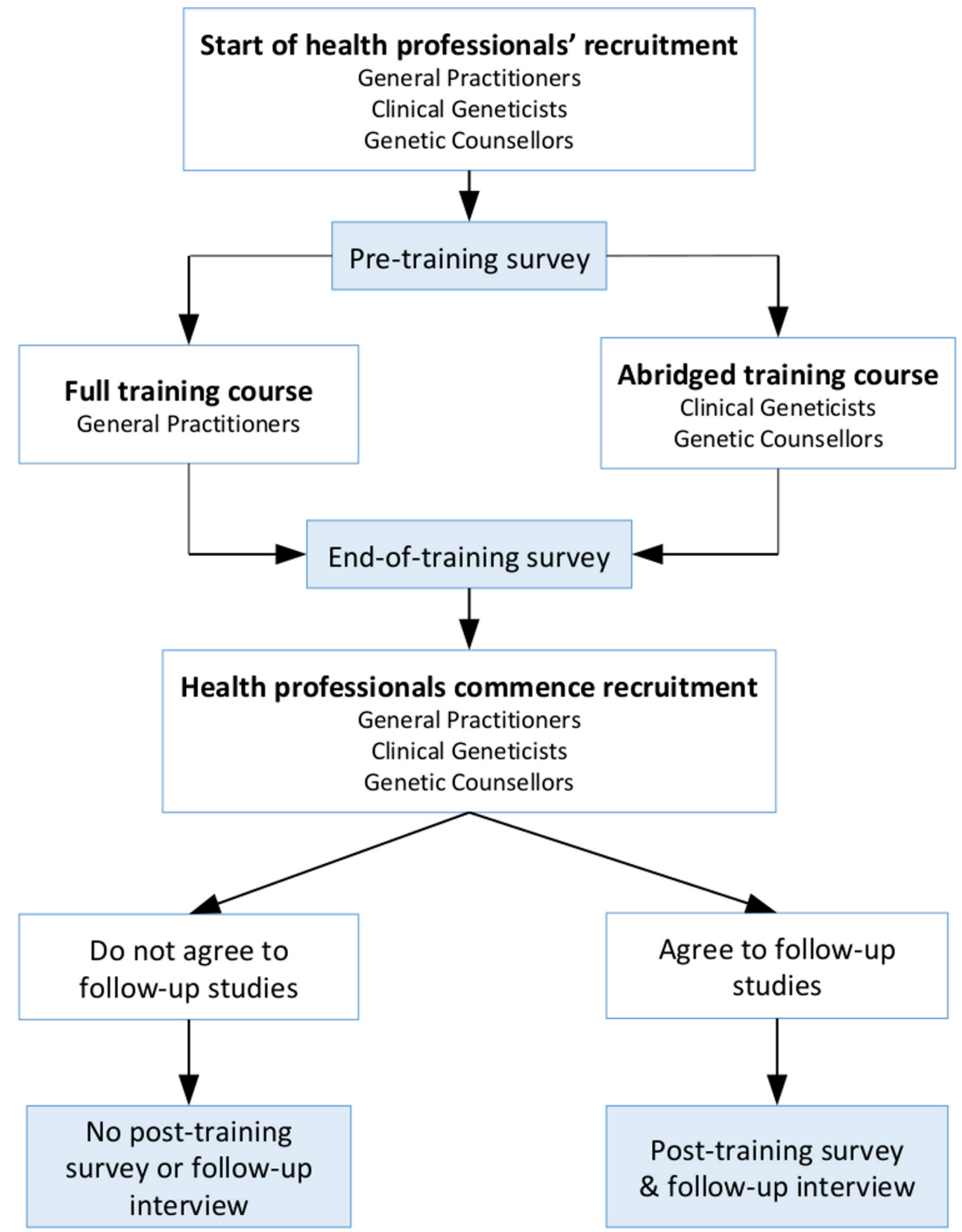

Figure 1 Health professionals were invited to participate in the study. Full training programme consists of aspects of genetic counselling and the technology used for the test and its limitations. Full training programme will be provided to health professionals who are not used to genetic counselling, such as general practitioners, while an abridged training programme will be provided to health professionals who are used to genetic counselling but require additional information on technology used and its limitations. Mixed-methods longitudinal study will be conducted, in which assessments are made at three time points through either questionnaires and/or semistructured interviews, with both participating couples and recruiters.

\section{EVALUATION AND ANALYSIS}

The evaluation of the study will include assessment of the technical aspects of the PCS programme, as well as determining the GP, clinical geneticist, GC, laboratory personnel and participant experience of the programme, and reviewing factors that affect uptake of the programme.

\section{Evaluation and anticipated publications}

The participating couples and health professionals will be asked to participate in follow-up studies involving a series of surveys and interviews (figures 1 and 2). All instruments (interview questions and questionnaire) can be found in the supplementary information.
Evaluating the delivery experience of PCS in the healthcare system

1. An end-to-end PCS programme in WA within the health system - an evaluative study:

- Evaluate turnaround time and result delivery for the metropolitan and regional sites.

- Evaluate resources developed during the pilot study, for example, websites, short videos explaining key study principals and the GP counselling syllabus.

- Evaluate the accuracy and diagnostic value of the targeted next generation sequencing (NGS) panel.

- Evaluate workload and challenges of providing counselling for metropolitan and regional WA. 


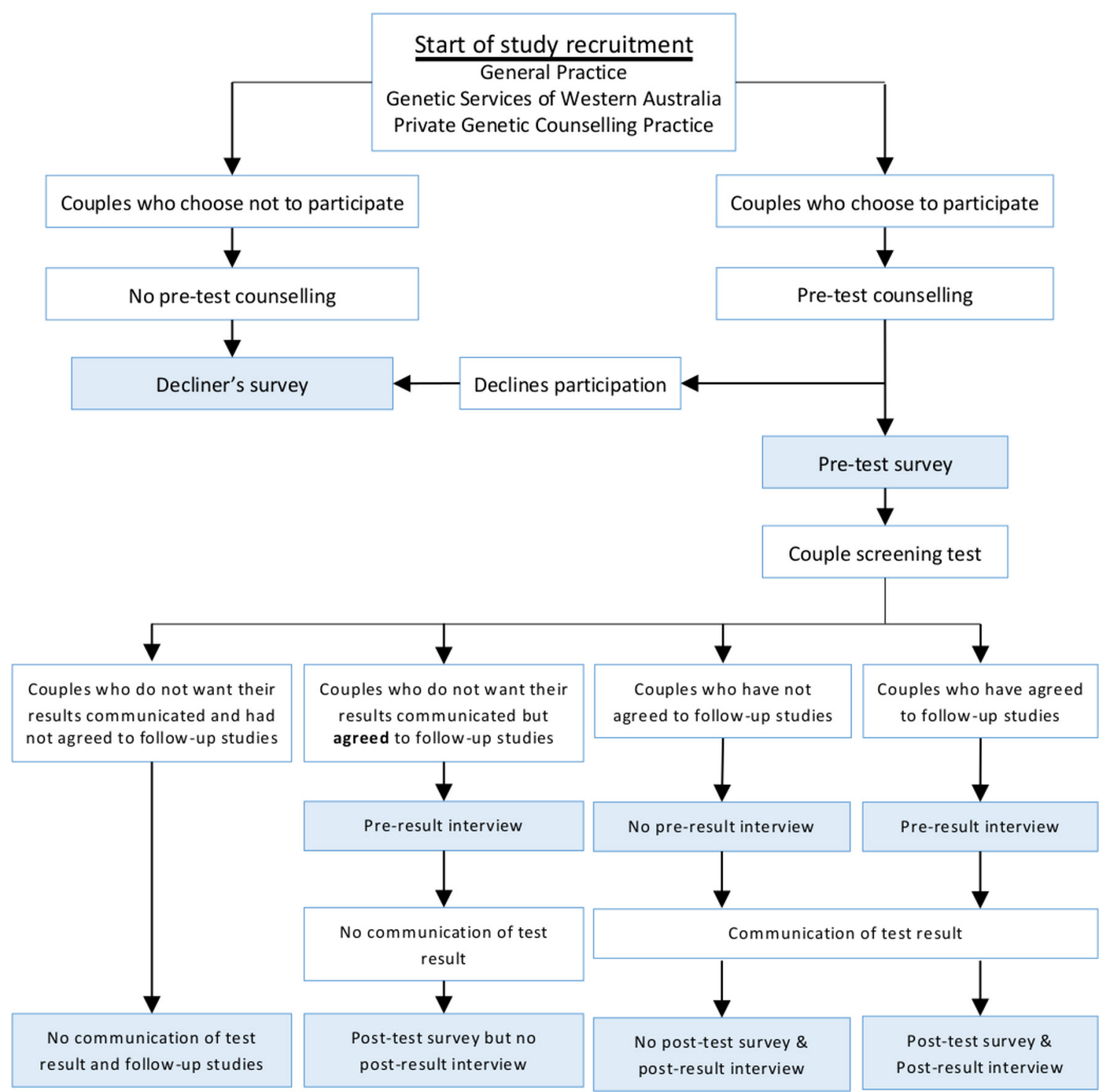

Figure 2 All recruiters will provide pretest counselling to couples interested in the preconception carrier-screening test. Couples who are interested in participating in the study will complete a pretest survey. Otherwise, the couple will complete a decliner's survey if they wish. Couples can choose to participate in follow-up studies and will receive a post-test survey, as well as participate in either a preresult or postresult interview. Mixed-methods longitudinal study will be conducted, in which assessments are made at three time points through either questionnaires and/or semistructured interviews, with both participating couples and recruiters.

- Evaluate workload and challenges of providing sequencing results for samples coming from metropolitan and regional WA.

- Evaluate problems faced during the pilot study and how they were resolved.

2. GPs' experience and challenges in providing pretest counselling for an expanded PCS programme in regional WA:

- Tools include GP pretest counselling education package, pretraining questionnaire end-of-training questionnaire and a follow-up post-training questionnaire plus telephone interview.

- Mix-method study evaluating the training syllabus for GPs and their experience, including limitations of the pilot study.
3. GC experience and challenges in providing pretest counselling for expanded PCS programme in metropolitan WA:

- Tools include telephone interview.

- Mix-method study evaluating GC experience, including limitations of the pilot study.

4. Implementing a state-wide PCS programme using next-generation sequencing technologies-outcomes and lessons learnt:

- Tools include a targeted MPS panel.

- Evaluate the targeted MPS panel for accuracy, including calling and identifying copy number variations using metrics such as Phred Score, call quality and average coverage. 
- Evaluate the number of couples at risk of having an affected child.

Evaluating factors that influence reproductive autonomy and uptake rates

5. Factors that influence informed decision making, anxiety, genetic knowledge and uptake rates of PCS test in WA

- Tools include participant pretest counselling questionnaire, posresult questionnaire and decliner questionnaire.

- This quantitative study will evaluate participant informed decision-making measured by the Multidimensional Measure of Informed Choice, as well as correlation studies between genetic knowledge level, informed decision-making and uptake rates. In addition, the content of the PCS panel will be evaluated with participants.

6. Participants' experience with a state-wide PCS programme

- Tools include participant preresult and postresult telephone interview.

- This qualitative study will evaluate participant experience by measuring their knowledge of the implications of the test, considerations, attitudes, whether they have deliberated before deciding and reproductive autonomy.

\section{Methods of analysis}

All participants with available data will be included in the study analyses.

A mixed-method strategy will be employed. Findings from interviews and surveys will guide the analysis to provide statistical information on the association between a particular behaviour and opinions or attitudes about the programme. Quotes from participants will be used to reinforce the correlation.

Descriptive statistics will be based on frequency distributions for categorical data and means, SD, CIs and ranges, or medians, IQR and ranges for continuous data, depending on normality. Univariate analysis will include $\chi^{2}$ and Fisher's exact tests, as appropriate, for categorical comparisons between groups, and t-tests and non-parametric Mann-Whitney U test for continuous outcomes. Categorical variables may be recoded into binary indicators if appropriate. Data will be analysed using a statistical software package such as IBM SPSS v25.0.0.1.

Thematic analysis will be used for interviews. The data generated will be managed using NVivo.

Probability values of $<0.05$ will be considered statistically significant.

\section{ETHICS AND DISSEMINATION}

This study, protocol and all instruments, including the informed consent document, have been approved by the Women and Newborn Health Service Human Research Ethics Committee (HREC) at King Edward Memorial
Hospital for Women (approval number RGS0000000946) and the University of Western Australia (UWA) HREC (approval number RA/4/20/4258).

\section{Withdrawal and handling of withdrawals}

Participants (health professional or couples) may choose to withdraw at any time for any reason. Withdrawal will in no way affect participating couples' current or future medical care. If participants (health professionals/ couples) withdraw, any of their data or samples that were collected will be kept, unless specifically requested to be destroyed.

If samples or data have been anonymised, it may not be possible to destroy them. Participants can notify the chief investigator, in writing, of their wishes in relation to the samples and data already collected. Withdrawn samples will be discarded in a timely manner.

\section{Assessment of safety}

The risks to the participants are those normally associated with drawing blood. It is possible that participants may feel some discomfort during the blood test and that there may be some bruising, swelling or bleeding where the needle enters the skin. Some people can feel a little light-headed when blood is taken.

Issues may arise that are associated with a couple, or family, knowing the potential cause of a disease, as opposed to the difficulties of not knowing the cause. All couples that receive a high-risk result will be referred for genetic counselling.

\section{Residual risk}

Residual risk will be addressed during pretest counselling and within the study information. Residual risks will exist, as some mutations may be undetected within the limitations of the test. A low-risk result does not mean that the couple has no chance of having a child with one of the screened conditions.

The PCS test also does not screen for all recessive genetic conditions, nor does it include dominantly inherited genetic conditions. This test also does not screen for chromosomal number or structural abnormalities or other health issues that may be identified in future offspring. Therefore, the risk of a couple having a child with these possibilities is not altered by PCS.

All participants will be able to contact the study GC for any clarification.

\section{Study closure}

The study will continue until target numbers have been recruited at each site.

When the study is concluded, all records from the study will be stored in a secure setting by the PI, Professor Nigel Laing, for a period of 10 years and then destroyed.

\section{Data collection}

The investigators are responsible for ensuring the accuracy, completeness, legibility and timeliness of the data reported. 


\section{Source data}

Source data will include DNA sequencing data and study-specific forms completed by the treating clinician or GC.

\section{Data capture methods}

Sequencing data will be captured and processed within the Department of Diagnostic Genomics, PathWest, according to NATA-accredited protocols.

All study-specific forms will have patient identifying details, but when entered into the study database, the patient will be identified only by the reidentifiable, study-specific unique identifier number assigned to each patient at enrolment. Evaluative data will be captured in both paper and electronic forms. Processed evaluative data will be transferred into a secure electronic database.

\section{Data storage}

All evaluation data will be deidentified and stored in a password-protected database in the UWA. In addition, all hard copy data collected (such as patient-specific forms) will be kept in a locked cabinet within a secure building. All electronic data will be stored in a password protected, backed up, location in the UWA Institutional Research Data Store.

DNA sequencing data will be stored by the Department of Diagnostic Genomics, PathWest, according to NATA protocols. The PathWest data will only be available to PathWest staff.

\section{Data and record retention}

All hard copy data (including consent forms and hard copy evaluation forms) will be stored in locked filing cabinets during the study. Data will be kept for a period of 10 years.

\section{Dissemination}

All results from the evaluative studies will be published.

\author{
Author affiliations \\ ${ }^{1}$ Centre for Medical Research, The University of Western Australia, Perth, Western \\ Australia, Australia \\ ${ }^{2}$ Molecular Medicine Division, Neurogenetic Diseases Laboratory, Harry Perkins \\ Institute of Medical Research, Nedlands, Western Australia, Australia \\ ${ }^{3}$ Genetic Services of Western Australia, King Edward Memorial Hospital for Women \\ Perth, Subiaco, Western Australia, Australia \\ ${ }^{4}$ PathWest Laboratory Medicine, Sir Charles Gairdner Hospital, Nedlands, Western \\ Australia, Australia \\ ${ }^{5}$ Illumina Inc, Scoresby, Victoria, Australia
}

\section{Acknowledgements The authors thank Ms Jocelyn Cleghorn and Ms Emma} Bourke for their role in the design of this pilot study.

Contributors RO, SE, DH, GR and NL designed the protocol. RO drafted the manuscript, coordinated the revisions and submitted the manuscript. R0, with the assistance of MD and MF, selected the genes for analysis. MF and MD designed the process for result analysis, comparison and reporting. $\mathrm{R} 0$, with the assistance of JB, designed the sample collection process for the study. BK, KH and NP provided feedback for couple recruitment in Genetic Services of Western Australia. All authors participated in the revision of the protocol. All authors commented on various versions of the manuscript, agreed on the final version to be published and can attest to the integrity of the work.
Funding Funding for the study for developing the next generation sequencing targeted gene panel for this project has been funded by the Perpetual Trustees Impact Philanthropy Program - 201601939. Funding has also been provided through Professor Laing's National Health and Medical Research Council Fellowship APP1117510 Translation Advancement Incentive and the Australian Genomics Health Alliance Project APP1113531 as well as philanthropic donations to Professor Laing's Neurogenetic Diseases Group in the Harry Perkins Institute of Medical Research including the Zac Pearson Legacy. RO is supported by an Australian Postgraduate Award and an Australian Genomics Health Alliance PhD top-up award (GNT1113531).

Disclaimer Funders of the study had no role in study design, data collection, data analysis, data interpretation or writing of the report.

Competing interests None declared.

Patient consent for publication Not required.

Provenance and peer review Not commissioned; externally peer reviewed.

Open access This is an open access article distributed in accordance with the Creative Commons Attribution Non Commercial (CC BY-NC 4.0) license, which permits others to distribute, remix, adapt, build upon this work non-commercially, and license their derivative works on different terms, provided the original work is properly cited, appropriate credit is given, any changes made indicated, and the use is non-commercial. See: http://creativecommons.org/licenses/by-nc/4.0/.

\section{REFERENCES}

1. Henneman L, Borry P, Chokoshvili D, et al. Responsible implementation of expanded carrier screening. Eur J Hum Genet 2017;25:1291.

2. Committee on Genetics. Committee Opinion No. 690: Carrier Screening in the Age of Genomic Medicine. Obstet Gynecol 2017;129:e35-e40.

3. Azimi M, Schmaus K, Greger V, et al. Carrier screening by nextgeneration sequencing: health benefits and cost effectiveness. $\mathrm{Mol}$ Genet Genomic Med 2016;4:292-302.

4. Schofield D, Alam K, Douglas L, et al. Cost-effectiveness of massively parallel sequencing for diagnosis of paediatric muscle diseases. NPJ Genom Med 2017;2.

5. Prior TW. Next-generation carrier screening: are we ready? Genome Med 2014;6:62.

6. Laing $\mathrm{N}$. What is the role of preconception carrier screening in neurology? Advances in Clinical Neuroscience and Rehabilitation 2016;16:2.

7. Plantinga M, Birnie E, Abbott KM, et al. Population-based preconception carrier screening: how potential users from the general population view a test for 50 serious diseases. Eur J Hum Genet 2016;24:1417-23.

8. Superior Health Council Belgium. Expanded carrier screening in a reproductive context. Towards a responsible implementation in the healthcare system. Belgium: Superior Health Council, 2017.

9. Ong R, Howting D, Rea A, et al. Measuring the impact of genetic knowledge on intentions and attitudes of the community towards expanded preconception carrier screening. J Med Genet 2018;55:744-52.

10. Holtkamp KCA, Mathijssen IB, Lakeman P, et al. Factors for successful implementation of population-based expanded carrier screening: learning from existing initiatives. Eur J Public Health 2017;27:372-7

11. BPMRI. Busselton Population Medical Research Institute - History. http://bpmri.org.au/about-us/history.html\#item-3 [Accessed 26 Nov 2018].

12. Edwards JG, Feldman G, Goldberg J, et al. Expanded carrier screening in reproductive medicine-points to consider: a joint statement of the American College of Medical Genetics and Genomics, American College of Obstetricians and Gynecologists, National Society of Genetic Counselors, Perinatal Quality Foundation, and Society for Maternal-Fetal Medicine. Obstet Gynecol 2015;125:653-62.

13. Richards S, Aziz N, Bale S, et al. Standards and guidelines for the interpretation of sequence variants: a joint consensus recommendation of the American College of Medical Genetics and Genomics and the Association for Molecular Pathology. Genet Med 2015;17:405-23.

14. Lazarin GA, Goldberg JD. Current controversies in traditional and expanded carrier screening. Curr Opin Obstet Gynecol 2016;28:136-41. 
15. Holtkamp KC, van Maarle MC, Schouten MJ, et al. Do people from the Jewish community prefer ancestry-based or pan-ethnic 